RELATO DE CASO

\title{
Aneurisma de artéria esplênica corrigido por embolização com molas
}

\author{
Splenic artery aneurysm treated by coil embolization \\ Rafaele Maria Araújo de Sena Pino', Eduardo Alexandre Souza Gois', Larissa Gouveia Aragão', Ângelo Mário de Sá Bomfim \\ Filho', David Campos Wanderley ${ }^{1}$
}

\begin{abstract}
Resumo
O aneurisma da artéria esplênica é uma entidade clínica rara, embora seja o mais frequente entre os aneurismas viscerais, sendo encontrado em 0,8\% da população. Apresenta-se mais frequentemente em mulheres, na proporção de 4:1, e raramente provoca sintomas ou sinais clínicos. Desenvolve-se de forma assintomática e, na maioria dos casos, é diagnosticado por meio de exames indicados para elucidar queixas clínicas decorrentes de outras doenças ou quando apresenta complicações por vezes fatais, como a rotura. A possibilidade de rotura dos aneurismas de artéria esplênica com diâmetro inferior a $2 \mathrm{~cm}$ é baixa; entretanto, os que apresentam diâmetro igual ou maior que $3 \mathrm{~cm}$ são usualmente encaminhados para tratamento cirúrgico, devido ao alto risco de rotura. O tratamento eletivo é indicado nos casos não complicados, sendo a embolização com molas um método interessante por evitar o tratamento cirúrgico convencional.
\end{abstract}

Palavras-chave: Artéria esplênica; doenças vasculares; embolização terapêutica.

\begin{abstract}
The splenic artery aneurysm is an uncommon clinical entity, but is the most frequent among visceral aneurysms and is present in $0.8 \%$ of the population. It is more common in women, with a ratio of 4:1, and rarely causes symptoms or signs. It develops asymptomatically and, in most cases, is diagnosed by tests indicated to elucidate clinical complaints subsequent to other diseases or when there are fatal complications such as rupture. The possibility of rupture of splenic artery aneurysms with a diameter less than $2 \mathrm{~cm}$ is low; however, those with a diameter equal to or greater than $3 \mathrm{~cm}$ are usually referred for surgical treatment due to the high risk of rupture. The elective treatment is indicated for non complicated cases, and the coil embolization is an interesting method, since it avoids conventional surgical treatment.
\end{abstract}

Keywords: Splenic artery; vascular diseases; embolization, therapeutic.

\section{Introdução}

O aneurisma da artéria esplênica é de ocorrência rara, embora seja o mais frequente entre os aneurismas viscerais ${ }^{1}$. Sua prevalência na população é em torno de $0,8 \%{ }^{2}$. O mecanismo de formação desse aneurisma ainda é desconhecido. Em relação ao seu diagnóstico, é frequentemente acidental e feito por meio de exames de imagem, solicitados para o diagnóstico de outras doenças. $\mathrm{O}$ tratamento cirúrgico eletivo está indicado para aneurismas a partir de $2 \mathrm{~cm}$ de diâmetro, que tenham crescimento rápido, ou caso se tornem sintomáticos no acompanhamento clínico, ou ainda quando associados com pancreatite e pseudocisto pancreático ${ }^{1}$.

\section{Relato de caso}

Mulher, 70 anos, hipertensa, procurou o serviço de nefrologia com queixa de infecção urinária de repetição. Após realização de uma tomografia computadorizada de abdômen, foi constatada a presença de três dilatações aneurismáticas em artéria mesentérica superior, renal direita e esplênica. A paciente foi encaminhada ao serviço de Angiologia. Foi realizada uma angiotomografia de abdômen, que também demonstrou dilatações aneurismáticas em artéria mesentérica superior, renal direita e esplênica (Figuras 1, 2 e 3). Realizou-se uma angiografia pré-operatória por cateterismo femoral retrógrado, destacando-se os seguintes achados: artéria mesentérica superior pérvia,

Trabalho realizado na Universidade Estadual de Ciências da Saúde de Alagoas (Uncisal), Maceió (AL), Brasil.

${ }^{1}$ Acadêmicos de Medicina da Universidade Estadual de Ciências da Saúde de Alagoas (Uncisal), Maceió (AL), Brasil.

${ }_{2}^{2}$ Médico; Radiologista Intervencionista da Fundação Hospital da Agro-Indústria do Açúcar e do Álcool de Alagoas, Maceió (AL), Brasil.

Não foram declarados conflitos de interesse associados à publicação deste artigo.

Fonte de fomento: nenhuma.

Submetido em: 26.10.2009 Aceito em: 08.12.2010

J Vasc Bras. 2010;9(4):249-253. 


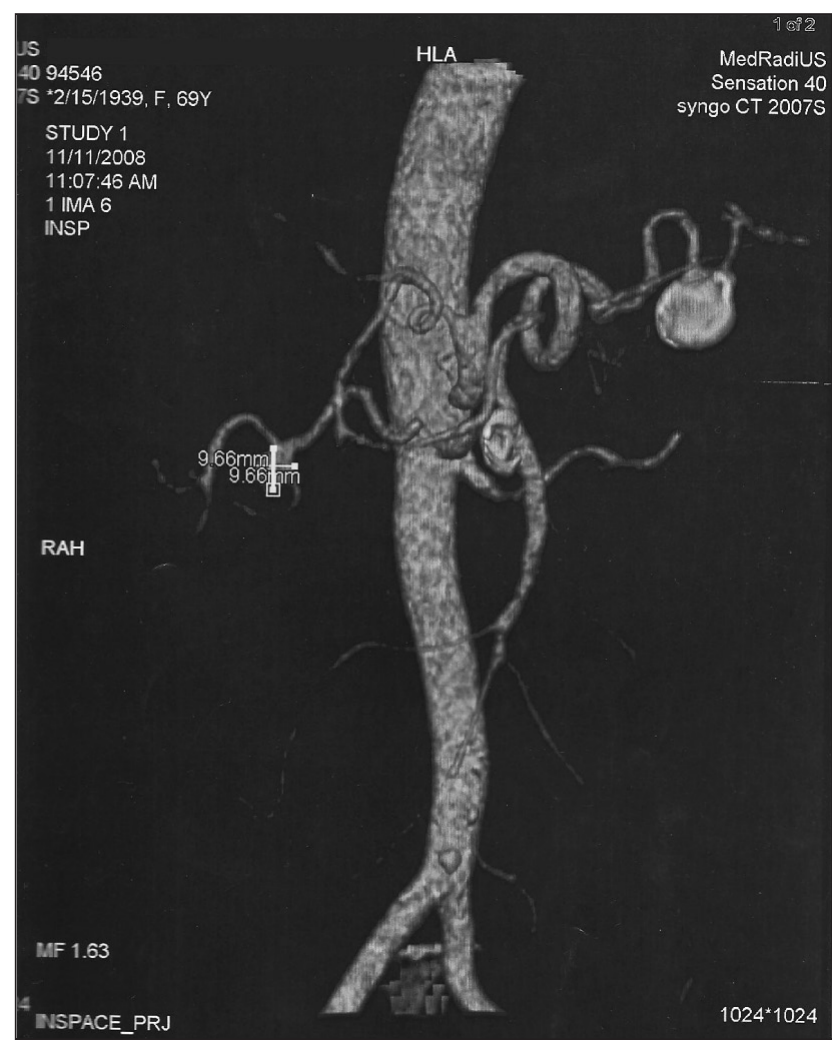

Figura 1 - Angiotomografia de aorta abdominal: dilatação aneurismática em artéria esplênica, artéria mesentérica superior e artéria renal direita opacificando as artérias gastroduodenal e hepática através da arcada pancreática, onde se observou pequeno aneurisma sacular, parcialmente calcificado, com sua porção pérvia medindo cerca de $0,4 \mathrm{~cm}$ (Figura 4); artéria renal direita pérvia, notando-se a presença de aneurisma sacular de colo largo, ao nível da sua bifurcação, medindo cerca de $1 \mathrm{~cm}$; artéria renal esquerda pérvia, dentro da normalidade; artéria esplênica pérvia, exibindo volumoso aneurisma parcialmente calcificado, de colo largo, em seu terço distal (extraparenquimatoso), medindo cerca de $2 \mathrm{~cm}$. O procedimento cirúrgico para correção do aneurisma foi marcado para três semanas após a realização da angiografia. Realizou-se punção femoral direita com cateterismo seletivo do tronco celíaco através de cateter Simons 2 e microcateterismo do aneurisma da artéria esplênica com microcateter Excelsior SL1018 (Boston Scientific, Natick, MA, USA), embolizado com 12 molas destacáveis de Guglielmi (GDC, do inglês Guglielmi detachable coils). As imagens de controle evidenciaram exclusão do aneurisma e perviedade dos demais ramos. Para os demais aneurismas, a conduta foi expectante, sendo acompanhados a cada seis meses para, se necessário, realizar o procedimento cirúrgico corretivo. A paciente recebeu alta hospitalar três dias após a realização do procedimento cirúrgico, permanecendo assintomática até o presente momento.

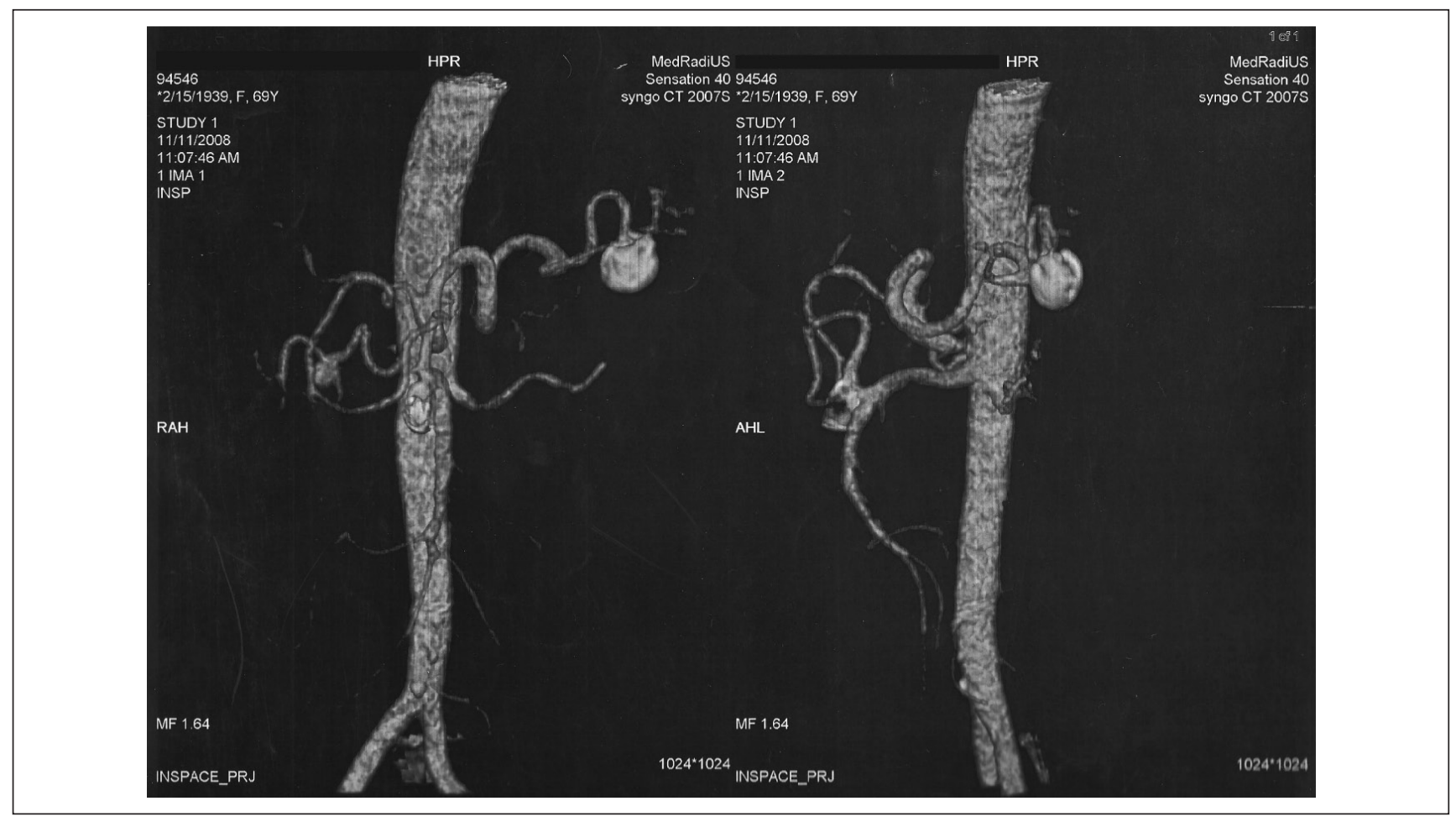

Figura 2 - Angiotomografia de aorta abdominal: dilatação aneurismática em artéria esplênica, artéria mesentérica superior e artéria renal direita 


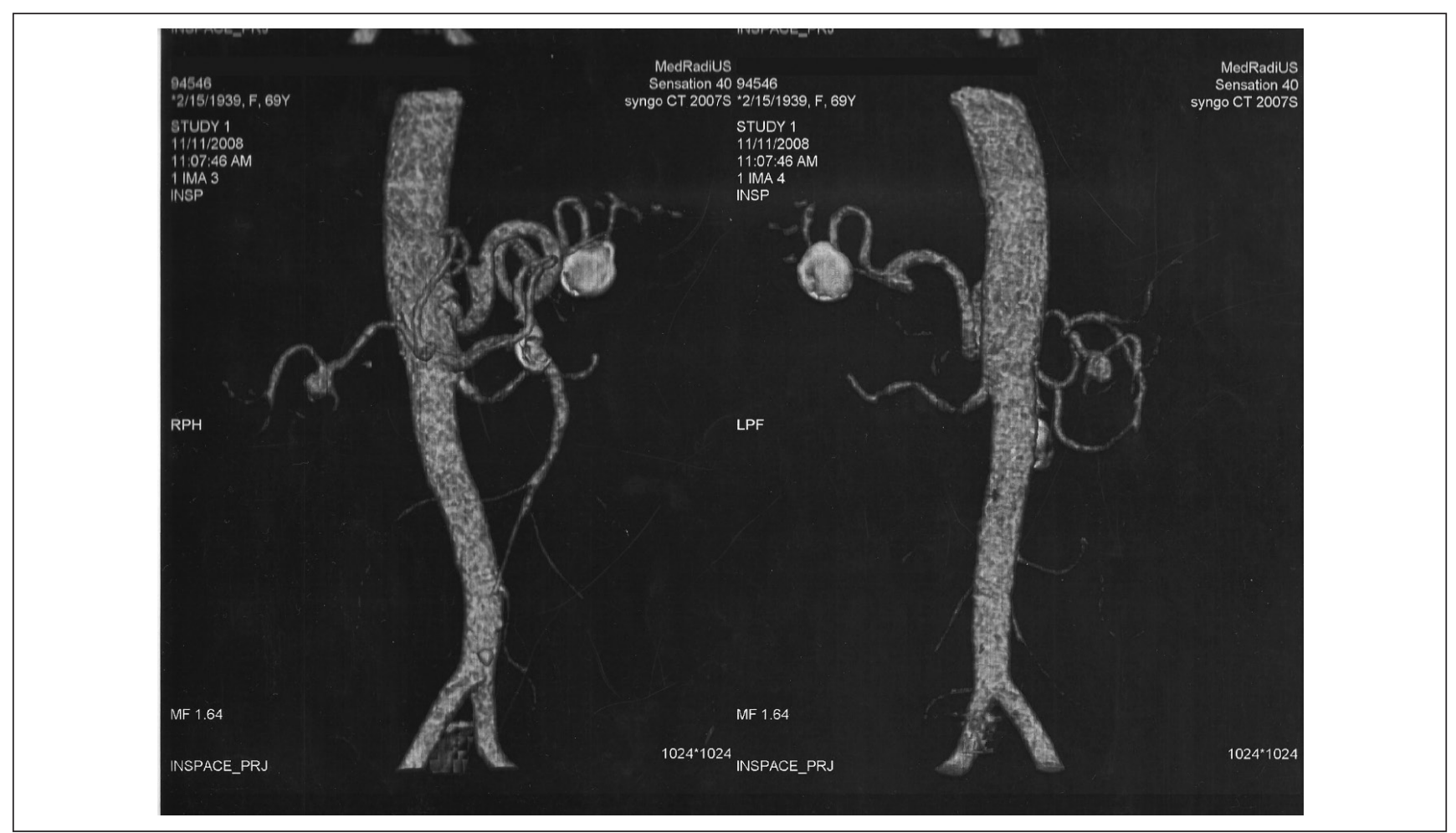

Figura 3 - Angiotomografia de aorta abdominal: dilatação aneurismática em artéria esplênica, artéria mesentérica superior e artéria renal direita

\section{Discussão}

O aneurisma da artéria esplênica (AAE) é uma entidade clínica rara, mas ainda assim é o terceiro entre as artérias abdominais e o mais frequente aneurisma visceral, correspondendo a $60 \%$ dos casos. É o mais frequente dos aneurismas esplâncnicos, sendo a prevalência na população ao redor de $0,8 \%{ }^{2}$, apresentando risco de ruptura aumentado em pacientes grávidas (devido ao aumento do fluxo esplênico e à alteração da elastina dos vasos) e em casos de hipertensão portal.

O mecanismo preciso da dilatação aneurismática da artéria esplênica é desconhecido. Ainda é atual a afirmação de Trimble e Hill ${ }^{3}$, que relataram dois fatores determinantes: fragilidade preexistente da parede arterial e aumento da pressão sanguínea.

Várias são as afecções relacionadas com o desenvolvimento de aneurismas da artéria esplênica, como aterosclerose, multiparidade, hipertensão portal, processos inflamatórios intra-abdominais, traumas abdominais, doenças do tecido conjuntivo, aneurismas congênitos ou êmbolos micóticos ${ }^{4-6}$.

Esse tipo de aneurisma é mais comum em mulheres, na proporção de 4:1. A maioria localiza-se no terço distal da artéria esplênica, podendo estar associado a outros aneurismas na mesma artéria ou em outros vasos ${ }^{5}$. A maioria dos aneurismas esplênicos é sacular e localiza-se próximo às bifurcações arteriais, podendo estar presente a calcificação parietal.

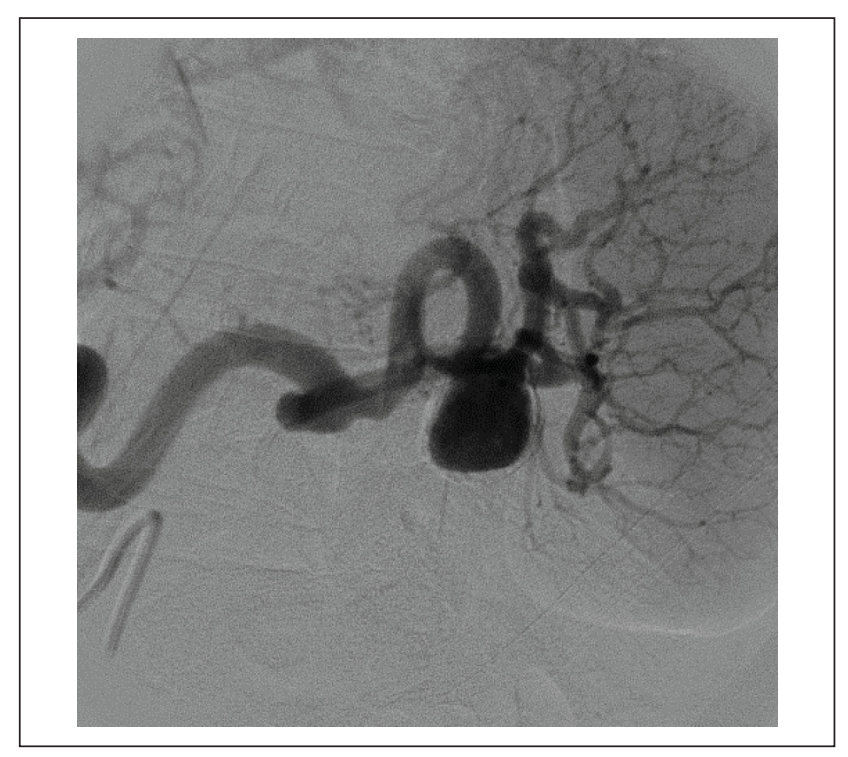

Figura 4 - Angiografia pré-operatória demonstrando dilatação aneurismática em artéria esplênica

Os primeiros relatos sugeriram um risco de ruptura em torno de $10 \%$. Atualmente, os estudos sugerem que esse risco foi superestimado e que as taxas de ruptura são em torno de 2 a $3 \%^{7-10}$. De qualquer forma, a ruptura ocorre e é mais comum em certos grupos de risco. Cerca de $50 \%$ das 
rupturas ocorrem durante a gravidez $\mathrm{z}^{11-13}$ e outros $20 \%$, em pacientes com hipertensão portal.

$\mathrm{O}$ AAE raramente provoca sintomas ou sinais clínicos, principalmente quando seu diâmetro não ultrapassa $2 \mathrm{~cm}^{14}$. É incomum o aparecimento de dor no quadrante superior esquerdo do abdome ou na região epigástrica. Entretanto, alguns pacientes relatam dor epigástrica crônica, inespecífica ou dor no hipocôndrio esquerdo. O aparecimento de dor aguda no quadrante superior esquerdo indica que a ruptura do aneurisma pode ter ocorrido, especialmente se estão presentes sinais de hipovolemia ${ }^{15}$.

Esse tipo de aneurisma não possui sinais clínicos característicos. É capaz de apresentar manifestações que, ocasionalmente, podem ser atribuídas a outras enfermidades - como é o caso dos sopros, que, quando presentes, possivelmente são correlacionados ao fluxo turbilhonar na aorta. O mesmo pode ocorrer no caso da presença de massa palpável no quadrante superior esquerdo, o que também pode ser devido a aneurisma esplênico; porém, é mais comum sua relação com uma esplenomegalia.

Por conta disso, a maioria dos casos não é diagnosticada até apresentar complicações por vezes fatais, nos casos das rupturas; o diagnóstico também é realizado por meio de exames indicados para pesquisa de outras doenças. A radiografia simples de abdome pode demonstrar a calcificação descrita como "anel de sinete", sugestivo desse aneurisma. Outros exames subsidiários também fazem o diagnóstico, como a ultrassonografia, a tomografia computadorizada ou a ressonância nuclear magnética ${ }^{16}$.

Embora o mapeamento dúplex seja um exame não invasivo, com mínimo risco para o paciente, é sabidamente examinador-dependente na visualização do AAE, e o exame radiológico com contraste acaba sendo necessário para confirmar o diagnóstico ${ }^{16}$. A tomografia computadorizada pode indicar o diagnóstico, sobretudo com uso de contraste iodado venoso; entretanto, a angiografia ainda é o método mais utilizado para diagnóstico e avaliação do AAE, além de avaliar melhor possíveis aneurismas de outras artérias viscerais ${ }^{17}$. Os recentes avanços da angiografia por ressonância magnética e tomografia computadorizada helicoidal auxiliarão no diagnóstico clínico mais acurado do $\mathrm{AAE}^{17}$.

O fácil acesso a exames pouco invasivos, como ultrassonografia, ressonância nuclear magnética e tomografia, vem aumentando o diagnóstico eletivo e incidental dos $\mathrm{AAE}^{18}$. Com o emprego crescente da arteriografia visceral, mapeamento dúplex e tomografia computadorizada, essas lesões estão sendo detectadas com maior frequência ${ }^{17}$.

A possibilidade de ruptura é bastante baixa para aneurismas com diâmetro inferior a 2 ou $3 \mathrm{~cm}$, exceção feita a gestantes e pacientes com hipertensão portal submetidos a procedimentos cirúrgicos como shunts portossistêmicos ou transplante hepático ${ }^{4-6}$.

O tratamento para aneurisma de artéria esplênica deve ser realizado quando se considera haver maior risco de rotura (diâmetro superior a $2 \mathrm{~cm}$ ), em doentes sintomáticos, transplantados, associados a processos inflamatórios e em mulheres em idade fértil ou grávidas ${ }^{19}$. Pacientes com AAE maiores que $3 \mathrm{~cm}$ são usualmente encaminhados para tratamento cirúrgico devido à possibilidade de ruptura, que aumenta com o diâmetro do mesmo ${ }^{4}$.

Existem várias abordagens cirúrgicas para o tratamento do aneurisma da artéria esplênica: ressecção, com ou sem reparação vascular, ligadura simples efetuada por via aberta ou laparoscópica e procedimentos endovasculares, como embolização. A via clássica, aberta ou por laparotomia, é um método de eficácia comprovada ${ }^{14}$. A via laparoscópica é uma alternativa excelente e pouco agressiva; entretanto, requer um cirurgião hábil em cirurgia endoscópica. O tratamento endovascular traz como dificuldades a colocação do próprio stent, pelo fato de a artéria esplênica ser tortuosa.

Os recentes avanços no diagnóstico por imagem têm fornecido subsídios fundamentais aos critérios anatômicos na escolha da técnica operatória a ser utilizada, e esta dependerá da localização anatômica do aneurisma e da anatomia vascular. Quando o aneurisma está localizado na porção distal da artéria, a correção pode ser realizada por operação aberta com ligadura, podendo ser necessária a esplenectomia ${ }^{19}$.

A embolização com coil é desaconselhável nos aneurismas gigantes pela ineficiência na oclusão do aneurisma, pelo intenso processo inflamatório que ocorre e pela possibilidade de embolização ${ }^{19}$. Apesar disso, a embolização percutânea com molas de Gianturco e gelfoam ${ }^{\circledast}$ (Pfizer, Inc.) tem sido relatada com resultados favoráveis. O método pode, muitas vezes, tornar desnecessária a intervenção cirúrgica ou ser associado ao tratamento cirúrgico, promovendo controle temporário das lesões, especialmente pseudoaneurismas, enquanto se alcança uma melhora das condições gerais do paciente que será submetido à cirurgia. A embolização pode ser especialmente vantajosa para pacientes que apresentam sangramento de aneurismas viscerais após drenagem cirúrgica de pseudocisto pancreático ${ }^{4} \mathrm{e}$ para pacientes com alto risco operatório devido à presença de comorbidades associadas ${ }^{20}$. Assim, a embolização seletiva - como primeira escolha terapêutica para o aneurisma sacular de artéria esplênica - vem se mostrando uma opção factível, devido à máxima preservação do parênquima esplênico com mínima morbidade ${ }^{21}$ e um período de internação hospitalar breve ${ }^{22}$. A técnica apresenta um sucesso na exclusão aneurismática de aproximadamente $85 \%{ }^{20}$. 
Como todo procedimento invasivo, a embolização não é isenta de complicações. A técnica está associada a um risco de oclusão do lúmen vascular com infarto esplênico e à recanalização da embolização, reportada em $4 \%$ dos ca$\operatorname{sos}^{20}$. Até $25 \%$ dos pacientes submetidos ao procedimento podem apresentar áreas de infarto esplênico, sendo esse risco maior quanto mais distal for a embolização ${ }^{22}$.

O tratamento eletivo do aneurisma de artéria esplênica é recomendado nos casos diagnosticados sem rotura. Devese levar em conta o risco cirúrgico da exérese, que deve ser menor que $0,5 \%$. Caso o risco operatório seja elevado, podese considerar a possibilidade de embolização percutânea do aneurisma ou mesmo a ligadura por via laparoscópica ${ }^{17,20}$. Embora alguns autores recomendem apenas correção do aneurisma nos doentes de baixo risco operatório ${ }^{23}$, estudos mais recentes concluem que um tratamento é determinado pelas dimensões do aneurisma, anatomia, risco de rotura e clínica apresentada pelo doente ${ }^{19}$.

A história natural dos aneurismas de artéria esplênica não é conhecida, pelo fato de serem assintomáticos e mais frequentemente diagnosticados na investigação de outra doença abdominal ou por ocasião da rotura, com alta mortalidade perioperatória. Por isso, apesar de serem pouco frequentes, devem ser tratados ao serem diagnosticados, desde que apresentem diâmetro superior a $2 \mathrm{~cm}$ ou estejam associados a condições agravantes: mulher em idade fértil, na gestação antes do terceiro trimestre, em doentes com hipertensão arterial sistêmica ou aneurisma em expansão.

\section{Referências}

1. Spencer Netto FAC, Damasceno F, Alencar CRP. Aneurisma roto de artéria esplênica: flagrante tomográfico de sangramento. Rev Col Bras Cir. 2002;29:119-21.

2. Trastek VF, Pairolero PC, Joyce JW, Hollier LH, Bernatz PE. Splenic artery aneurysms. Surgery. 1982;91:694-9.

3. van Berge Henegouwen DP. Aneurysms of intestinal arteries. Langenbecks Arch Chir Suppl II Verh Dtsch Ges Chir. 1990:341-6.

4. Mattar SG, Lumsden AB. The management of splenic artery aneurysms: experience with 23 cases. Am J Surg. 1995; 169:580-4.

5. de Perrot M, Bühler L, Deléaval J, Borisch B, Mentha G, Morel P. Management of true neurysms of splenic artery. Am J Surg. 1998; 175:466-8.

6. Wagner $\mathrm{WH}$, Alexander AD, Treiman RL, et al. Ruptured visceral artery aneurysms. Ann Vasc Surg. 1997;11:342-7.

7. Evans GH, Gunn J, Castleden WM. Spontaneous rupture of a splenic artery aneurysm. Aust N Z J Surg. 1992;62:664-5.

8. Fischer $M$. Ruptured aneurysm of the lienalis artery as a cause of acute abdomen. Case report and review of the literature. Helv Chir Acta. 1991;58:359-63.
9. Seiler C, Blumgart LH. Gastrointestinal hemorrhage due to splenic artery aneurysm pancreatic duct fistula in chronic pancratitis. A case report and review of the literature. HPB Surg. 1993;7:149-55.

10. Wagner WH, Cossman DV, Treiman RL, Foran RF, Levin PM, Cohen JL. Hemosuccus pancreaticus from intraductal rupture of a primary splenic artery aneurysm. J Vasc Surg. 1994;19:158-64.

11. Angelakis EJ, Bair WE, Barone JE, Lincer RM. Splenic artery aneurysm rupture during pregnancy. Obstet Gynecol Surv. 1993;48:145-8.

12. Caillouette JC, Merchant EB. Ruptured splenic artery aneurysm in pregnancy. Twelfth reported case with maternal and fetal survival. Am J Obstet Gynecol. 1993;168:1810-1.

13. Hong GS, Wong CY, Nambiar R. Massive lower gastrointestinal hemorrhage from a splenic artery pseudoaneurysm. Br J Surg. 1992;79:174.

14. Zelenock GB, StanleyJC. Splachnic artery aneurysms. In: Rutherford RB, editor. Vascular surgery. 5th ed. Philadelphia: WB Saunders; 2000. p. 1369-82.

15. Larson PA, Lipchik EO, Adams MB. Development and regression of visceral artery aneurysms following liver transplantation: case report. Cardiovasc Intervent Radiol. 1988;11:75-8.

16. Miranda Júnior F. Aneurismas vicerais e periféricos. In: Pitta GBB, Castro AA, Burihan E, editores. Angiologia e cirurgia vascular: guia ilustrado. Maceió: UNCISAL/ECMAL \& LAVA; 2003. p. 1-10.

17. Smith JA, Macleish DG, Collier NA. Aneurysms of the visceral arteries. Aust N Z J Surg. 1989;59:329-34.

18. Salam TA, Lumsden AB, Martin LG, Smith RB 3rd. Nonoperative management of visceral aneurysms and pseudoaneurysms. Am J Surg. 1992;164:215-9.

19. Guillaumon AT, Chaim EA. Splenic artery aneurysm associated with anatomic variations in origin. J Vasc Bras. 2009;8:177-81.

20. Mandolfino T, Canciglia A, D'Alfonso M. Aneurismi dell'arteria splenica. Nostra esperienza. Chirurgia Italiana. 2009;61:315-9.

21. Halloul Z, Meyer F, Grote R, Lippert H, Buerger T. Selective embolization of splenic artery aneurysm - case report. Eur Surg. 2005;37:59-62.

22. Larraín D, Fava M, Espinoza R. Aneurisma de la arteria esplénica. Diagnóstico diferencial y alternativas terapéuticas. Rev Méd Chile 2005;133:943-6.

23. Tochii $M$, Ogino $H$, Sasaki $H$, et al. Successful surgical treatment for aneurysm of splenic artery with anomalous origin. Ann Thorac Cardiovasc Surg. 2005;11:346-9.

Correspondência Rafaele Maria Araújo de Sena Pino Rua Antonio Menezes de Araujo Lemos, n॰ 39 - Gruta de Lourdes CEP 57052-725 - Maceió (AL), Brasil E-mail: faele.sena@hotmail.com

Contribuições dos autores: Concepção e desenho do estudo: AMSBF, EASC, RMASP e LGA Análise e interpretação dos dados: RMASP e LGA Coleta de dados: RMASP, LGA e DCW Redação do artigo: EASG Revisão crítica do texto: AMSBF e EASG Aprovação final do artigo*: RMASP, EASG, AMSBF, LGA e DCW Análise estatística: N/A

Responsabilidade geral pelo estudo: RMASP, EASG, AMSGF e LGA *Todos os autores leram e aprovaram a versão final submetida ao J Vasc Bras. 